\title{
The Effect of Strong Near Surface Scattering on Seismic Imaging: Investigation Based on Resolution Analysis
}

\author{
Xiao-Bi Xie ${ }^{1, *}$, Baoqing $\mathrm{He}^{1,2}$, Hongxiao $\mathrm{Ning}^{2}$, Yongqing $\mathrm{He}^{2}$ and \\ Bo Chen ${ }^{3}$ \\ ${ }^{1}$ Institute of Geophysics and Planetary Physics, University of California at Santa \\ Cruz, CA 95064, USA. \\ ${ }^{2}$ China National Petroleum Corp Bureau of Geophysical Prospecting Inc., Center for \\ Acquisition Technology, Zhuozhou, Hebei, China. \\ ${ }^{3}$ China School of Earth Science and Geological Engineering, Sun Yat-Sen University, \\ Guangzhou, Guangdong, China.
}

Received 27 March 2018; Accepted (in revised version) 19 January 2019

\begin{abstract}
In land seismic exploration, strong near-surface heterogeneities can cause serious problems in seismic data acquisition and the quality of depth imaging. By introducing random velocity models to simulate velocity fluctuations in the near-surface layer and using the point spread function to characterize image quality, we examine how the scattering generated in near-surface heterogeneities can affect the subsurface image. In addition to the commonly known scattering noises which lower the signal to noise ratio in seismic data, our results also reveal that intermediate scale heterogeneities generate forward scattering which forms phase or travel time fluctuations. Due to intermediate-scale uncertainty in the shallow part of the migration velocity model, these phase changes are carried to the target by the extrapolated wavefields, breaking the zero phase image condition at the image point. This is a primary reason for deteriorated image quality in regions with strong near-surface scattering. If this intermediate-scale information can be obtained and built into the migration velocity, the subsurface image quality can be largely improved. These results can be the basis for further numerical investigations and field experiments. The proposed analysis method can also be used to evaluate other potential methods for dealing with nearsurface scattering.
\end{abstract}

AMS subject classifications: 74J20, 86A15

Key words: Reverse time migration, shallow scattering, velocity model, prestack depth imaging, seismic resolution.

${ }^{*}$ Corresponding author. Email addresses: xxie@ucsc.edu (X.-B. Xie), hebaoqing@cnpc.com.cn (B. He), ninghongxiao@cnpc.com.cn (H. Ning), heyongqing@cnpc.com.cn (Y. He), bchen019@outlook.com (B. Chen) 


\section{Introduction}

Wave equation based prestack migration plays an important role in modern seismic imaging and has gained wide application in oil and gas exploration. However, in land exploration, particularly in certain regions in western China and the Middle East, the nearsurface layer is often composed of highly complicated small-scale low-velocity structures (e.g., rough topography, loess, deserts, strongly weathered layers, alluvium). Strong scattering and heavy attenuation generated in this layer bring serious difficulties to seismic acquisition and data processing [1-4]. Illustrated in Fig. 1 is a typical shot record in a region with strong shallow scattering in western China, where deep reflections are almost completely buried in the noise. Many processes are involved in near surface scattering. Because the earth is intrinsically elastic, there are both P-to-P and P-to-S scattered waves, causing reverberations in the shallow low-velocity layer. The undersampling due to sparse acquisition further complicates this process. Many authors have pointed out these effects and investigated related phenomena in either elastic or acoustic models [2,4]. For decades, researchers tested various techniques to mitigate this problem. Based on the assumption that scattering produces random noise, most techniques attempted to suppress scattering effects by stacking, e.g., grouped geophones, long survey lines, wide-line profiling and high-density acquisitions [5-7]. Although these techniques achieved limited successes in certain regions, the problems have not been satisfactorily solved in most cases, particularly in regions with very thick gravel or rough terrain [8].

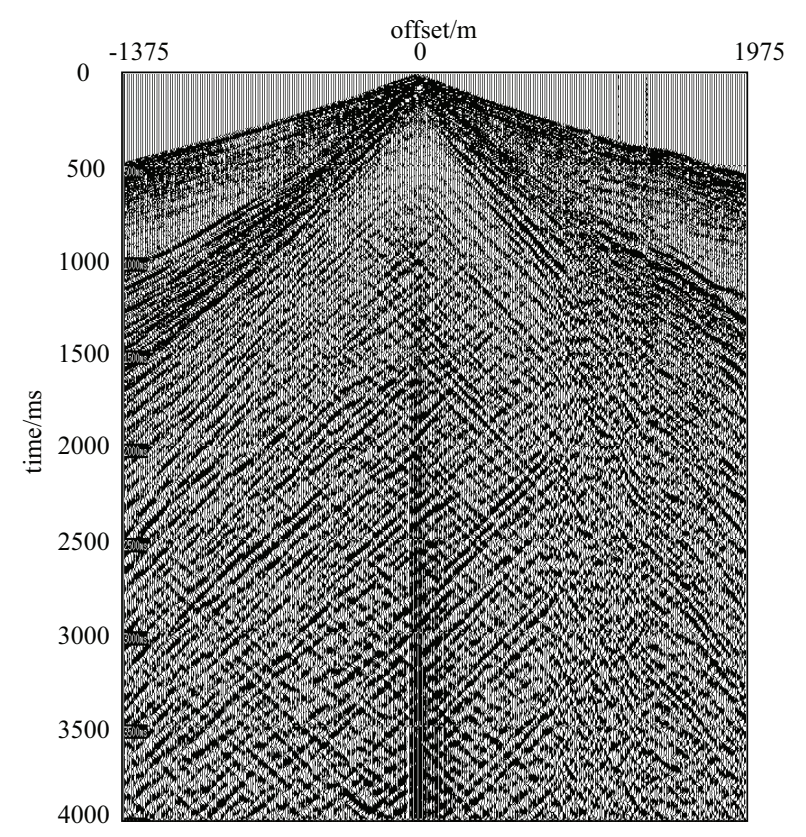

Figure 1: A shot record in a region with strong shallow scatterings in western China. 


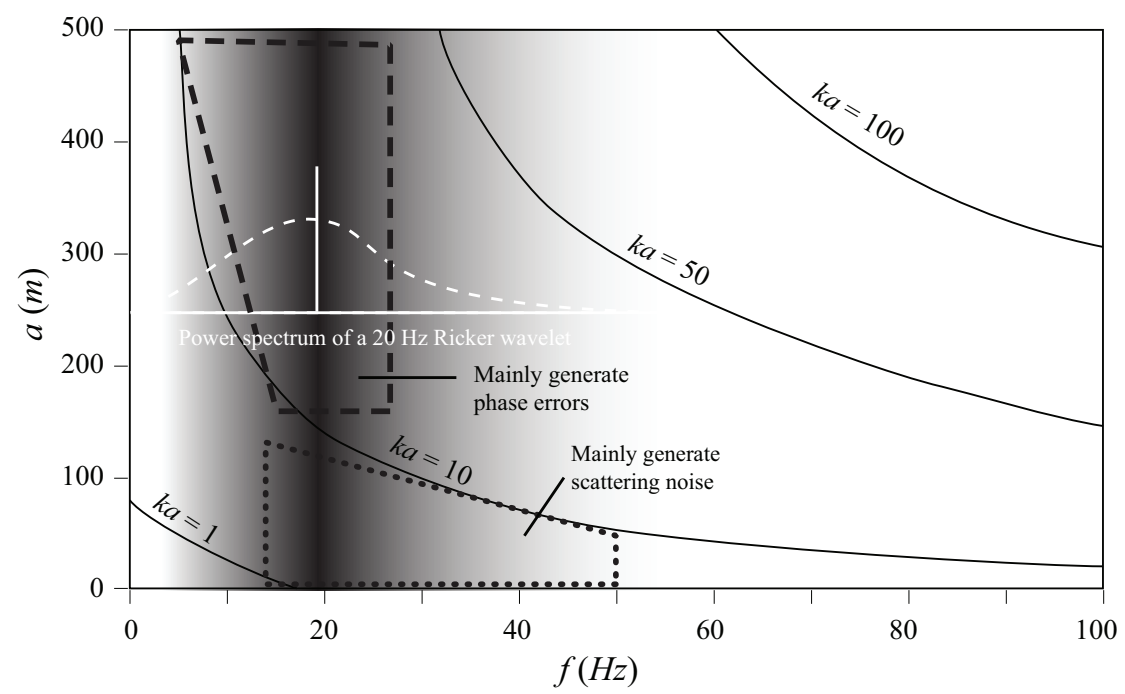

Figure 2: $k a$ values versus wave frequency $f$ and scatterer size $a . k a=10$ separates the large and small angle scattering regimes. The area enclosed by dotted line mainly generates scattered noise, and area enclosed by dashed line mainly generates phase errors. The shaded area is the power spectrum of a $20 \mathrm{~Hz}$ Ricker wavelet.

The typical scales of scatterers involved in these processes are from a few meters to several hundred meters, and their burial depths are usually less than $0.5-1 \mathrm{~km}$. They are often called "small-scale heterogeneities" because their scales are much smaller than those can be built into the migration velocity model. However, these heterogeneities still cover a wide-range of scales, and scatterers of different sizes affect wave propagation in quite different ways. The ratio between the size of the scatter and the wavelength, or the characteristic scale $k a$, where $k=2 \pi / \lambda$ is the wavenumber, $\lambda$ is the wavelength, and $a$ is the size of the scatterer, dominates the behavior of the scattering. Illustrated in Fig. 2 are $k a$ values in a model of $2000 \mathrm{~m} / \mathrm{s}$, and for wave frequency $f$ between 0 and $100 \mathrm{~Hz}$ and $a$ between 0 and $500 \mathrm{~m}$. Based on the scattering theory [9,10], when $k a \approx 1$ (e.g., $0.1<k a<10$ ), scatterers tend to deflect waves to larger scattering angles and move energy from primary arrivals to coda waves. Because seismic migration normally assumes primary reflections, large-angle scattering both weakens effective signals and introduces strong noise, causing low signal-to-noise ratios in data. On the other hand, when $k a \gg 1$, the intermediate to larger scale heterogeneities tend to generate small angle (forward) scattering, mainly causing travel time or phase errors. The actual seismic signal is broadband. The shadowed area in Fig. 2 represents the power spectrum of a $20 \mathrm{~Hz}$ Ricker wavelet. Taking the line $k a=10$ as a border to separate large/small angle scattering regimes, the $a$ values above this line tend to cause travel time errors (area enclosed by dashed line); while those below this line tend to generate scattering noise. Since the amplitude of scattered wave is proportional to $f^{2}$, the high frequency wave contents below $k a=10$, when interacting with very small-scale scatterers, are a major source for strong scattered noise (area enclosed by dotted line). Therefore, the scattering behavior of a broadband signal 
is jointly controlled by the frequency content of the wave and the scale distribution of the shallow heterogeneity. The interactions generate both scattering noise and cause phase errors. Stacking techniques may be effective for eliminating the scattering noise but not for removing phase errors.

The current study serves two purposes. First, we propose a method to investigate how shallow scattering affects the depth image. A random velocity layer is adopted to simulate the shallow velocity structure, and the point spread function is used to characterize the quality of the seismic depth image. Seismic imaging using reverse time migration (RTM) is used to connect the shallow random model to the depth image. The random models can be characterized using a small number of parameters, and point spread functions can be analyzed in either space or wavenumber domains. RTM does not have limitations on complex velocity models. Jointly, they provide a concise and highly flexible approach with which various techniques for solving surface scattering problems can be evaluated. Our second purpose was to investigate how shallow heterogeneities with different scales affect the image quality. Since scatterers of different scales affect the imaging process differently, we separated the near surface heterogeneities into three subcategories - small $(<100 \mathrm{~m})$, intermediate $(100-300 \mathrm{~m})$ and large $(300-500 \mathrm{~m}$ and above) - to investigate their contributions to the imaging process assuming typical seismic frequencies and wave speeds. In the rest of this paper, we will use these definitions of scales unless otherwise indicated.

\section{The seismic image and its resolution}

Considering a survey system composed of a source at $\mathbf{x}_{s}$ and a receiver at $\mathbf{x}_{g}$ to image a target region $V(\mathbf{x})$ located near $\mathbf{x}$ (refer to Fig. 3), under the Born approximation acoustic seismic data recorded at $\mathbf{x}_{g}$ can be expressed as

$$
D\left(\mathbf{x}, \mathbf{x}_{g}, \mathbf{x}_{s}, \omega\right)=2 s(\omega) \int_{V(\mathbf{x})} k_{0}^{2} G_{D}\left(\mathbf{x}^{\prime}, \mathbf{x}_{s}, \omega\right) M\left(\mathbf{x}^{\prime}\right) G_{D}\left(\mathbf{x}^{\prime}, \mathbf{x}_{g}, \omega\right) d \mathbf{x}^{\prime},
$$

where $\omega$ is the frequency, $\mathbf{x}^{\prime}$ is the location of a scatterer inside $V(\mathbf{x}), k_{0}=\omega / c_{0}(\mathbf{x})$ is the local wavenumber, $c_{0}(\mathbf{x})$ is the background velocity, $\delta c\left(\mathbf{x}^{\prime}\right)$ is the velocity perturbation, $M\left(\mathbf{x}^{\prime}\right)=\delta c\left(\mathbf{x}^{\prime}\right) / c_{0}(\mathbf{x})$ is the relative velocity perturbation, and $s(\omega)$ is the source spectrum. $G_{D}$ is the frequency domain Greens function, which represents either the actual propagation of waves in the real earth, or, in a synthetic case, the Greens function calculated in the true velocity model. $G_{D}\left(\mathbf{x}^{\prime}, \mathbf{x}_{s}, \omega\right)$ propagates the wave from the source to the target, and $G_{D}\left(\mathbf{x}^{\prime}, \mathbf{x}_{g}, \omega\right)$ propagates the scattered wave from the target to the receiver. Acoustic reciprocity $G_{D}\left(\mathbf{x}^{\prime}, \mathbf{x}_{g}, \omega\right)=G_{D}\left(\mathbf{x}_{g}, \mathbf{x}^{\prime}, \omega\right)$ is used. For an acquisition system composed of multiple sources and receivers, the depth image at $\mathbf{x}^{\prime \prime}$ in the vicinity of $\mathbf{x}$ can be expressed as

$$
I\left(\mathbf{x}, \mathbf{x}^{\prime \prime}, \omega\right)=s(\omega) \sum_{x_{s}} \sum_{x_{g}} G_{M}\left(\mathbf{x}^{\prime \prime}, \mathbf{x}_{s}, \omega\right) D^{*}\left(\mathbf{x}, \mathbf{x}_{g}, \mathbf{x}_{s}, \omega\right) \frac{\partial G_{M}\left(\mathbf{x}^{\prime \prime}, \mathbf{x}_{g}, \omega\right)}{\partial z_{g}},
$$




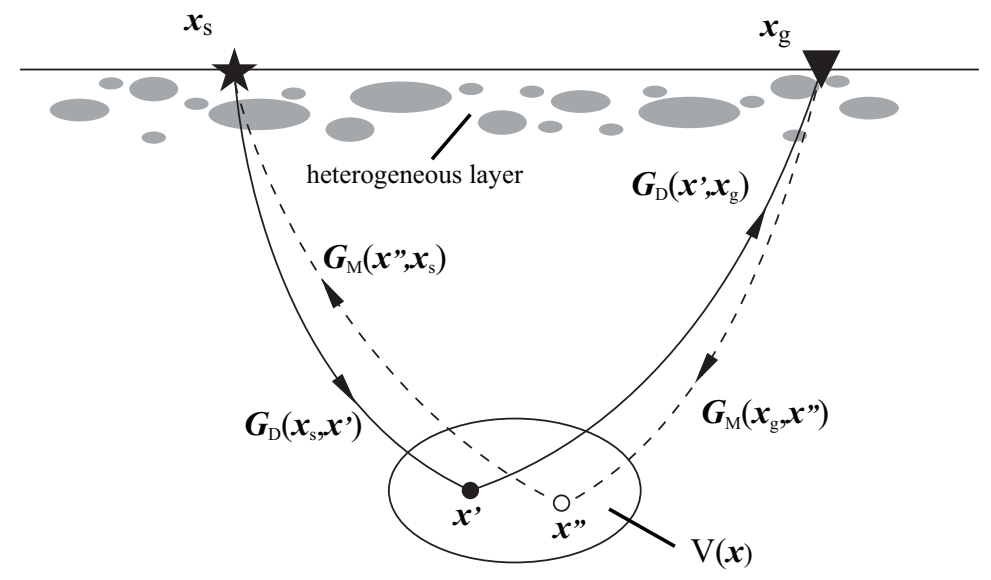

Figure 3: Cartoon showing the image process and the coordinate system.

where $G_{M}$ is the Greens function for migration calculated using the migration velocity model, $z_{g}$ is the $z$-component of $\mathbf{x}_{g}$. Eq. (2.2) forward propagates the source energy and back propagates the time reversed data to generate the image. The superscript "** denotes the complex conjugate. Substituting Eq. (2.1) into (2.2), the depth image can be expressed as (see e.g., [11,12])

$$
I\left(\mathbf{x}, \mathbf{x}^{\prime \prime}, \omega\right)=\int_{V(\mathbf{x})} M\left(\mathbf{x}^{\prime}\right) R\left(\mathbf{x}, \mathbf{x}^{\prime}, \mathbf{x}^{\prime \prime}, \omega\right) d \mathbf{x}^{\prime},
$$

where

$$
\begin{aligned}
R\left(\mathbf{x}, \mathbf{x}^{\prime}, \mathbf{x}^{\prime \prime}, \omega\right)= & 2 k_{0}^{2} s(\omega) s^{*}(\omega) \\
& \times \sum_{x_{s}} \sum_{x_{g}} G_{M}\left(\mathbf{x}^{\prime \prime}, \mathbf{x}_{s}, \omega\right) G_{D}^{*}\left(\mathbf{x}^{\prime}, \mathbf{x}_{s}, \omega\right) G_{D}^{*}\left(\mathbf{x}^{\prime}, \mathbf{x}_{g}, \omega\right) \frac{\partial G_{M}\left(\mathbf{x}^{\prime \prime}, \mathbf{x}_{g}, \omega\right)}{\partial z_{g}} .
\end{aligned}
$$

The function $R\left(\mathbf{x}, \mathbf{x}^{\prime}, \mathbf{x}^{\prime \prime}\right)$, through both forward and backward propagations, maps the velocity perturbation at $\mathbf{x}^{\prime}$ to the image at $\mathbf{x}^{\prime \prime}$. Therefore, $R\left(\mathbf{x}, \mathbf{x}^{\prime}, \mathbf{x}^{\prime \prime}\right)$ is also known as the point spread function (PSF), which is the response of the imaging system to a point scatterer at $\mathbf{x}^{\prime}$ (see e.g., [11-16]). Introducing the local Fourier transform over local variables $\mathbf{x}^{\prime}$ and $\mathbf{x}^{\prime \prime}$ in the neighborhood of $\mathbf{x}$, Eq. (2.3) can be converted into the wavenumber domain $\mathbf{k}_{d}[11,12]$

$$
I\left(\mathbf{x}, \mathbf{k}_{d}, \omega\right)=R\left(\mathbf{x}, \mathbf{k}_{d}, \omega\right) \cdot M\left(\mathbf{x}, \mathbf{k}_{d}\right),
$$

where

$$
\begin{aligned}
& M\left(\mathbf{x}, \mathbf{k}_{d}\right)=\int_{V(\mathbf{x})} M\left(\mathbf{x}^{\prime}\right) e^{i \mathbf{k}_{d} \cdot \mathbf{x}^{\prime}} d \mathbf{x}^{\prime} \\
& R\left(\mathbf{x}, \mathbf{k}_{d}, \omega\right)=2 k_{0}^{2} s(\omega) s^{*}(\omega) \sum_{\mathbf{x}_{s}} \sum_{\mathbf{x}_{g}} A\left(\mathbf{x}, \mathbf{k}_{d} ; \mathbf{x}_{s}, \mathbf{x}_{g}, \omega\right),
\end{aligned}
$$


and

$$
A\left(\mathbf{x}, \mathbf{k}_{d}, \mathbf{x}_{s}, \mathbf{x}_{g}, \omega\right) \stackrel{\mathbf{k}_{d}=\mathbf{k}_{s}+\mathbf{k}_{g}}{\longleftarrow} G_{M}\left(\mathbf{x}, \mathbf{k}_{s}, \mathbf{x}_{s}, \omega\right) G_{D}^{*}\left(\mathbf{x}, \mathbf{k}_{s}, \mathbf{x}_{s}, \omega\right) G_{D}^{*}\left(\mathbf{x}, \mathbf{k}_{g}, \mathbf{x}_{g}, \omega\right) \frac{G_{M}\left(\mathbf{x}, \mathbf{k}_{g}, \mathbf{x}_{g}, \omega\right)}{\partial z_{g}},
$$

where $\mathbf{k}_{d}=\mathbf{k}_{s}+\mathbf{k}_{g}$, and $\mathbf{k}_{s}$ and $\mathbf{k}_{g}$ are incident and scattered wavenumbers, and $R\left(\mathbf{x}, \mathbf{k}_{d}, \omega\right)$ is the wavenumber domain PSF. The mapping from $\left(\mathbf{k}_{s}, \mathbf{k}_{g}\right)$ to $\mathbf{k}_{d}$ in Eq. (2.8) converts the response from acquisition coordinate $\left(\mathbf{k}_{s}, \mathbf{k}_{g}\right)$ to target wavenumber coordinate $\mathbf{k}_{d}$.

From Eqs. (2.3) or (2.5), the image process near location $\mathbf{x}$ can be expressed as the convolution between $R\left(\mathbf{x}, \mathbf{x}^{\prime}, \mathbf{x}^{\prime \prime}\right)$ and the velocity perturbation $M\left(\mathbf{x}^{\prime}\right)$. Therefore, the PSF is composed of information regarding the quality of an imaging system. Note, in the above equations, an additional variable $\mathbf{x}$ emphasizes the analysis is localized, i.e., the PSF itself varies from location to location. Previous investigations $[11,12,15,16]$ all assumed the accurate migration velocity model is available, i.e., $G_{M}=G_{D}$. Under this circumstance, the PSF only reflects errors from uneven illumination. However, in real situations, the migration velocity model has errors compared to the true velocity. To introduce this error, we assume that

$$
G_{M}\left(\mathbf{x}, \mathbf{k}_{s}, \mathbf{x}_{s}, \omega\right)=a_{s} e^{i \omega \delta \tau_{s}} G_{D}\left(\mathbf{x}, \mathbf{k}_{s}, \mathbf{x}_{s}, \omega\right),
$$

where the factor $a_{s} e^{i \omega \delta \tau_{s}}$ is the error caused by an inaccurate migration velocity model, $a_{s}=\left|G_{M} / G_{D}\right|$ is the amplitude error, $\omega \delta \tau_{s}=\arg \left(G_{M} / G_{D}\right)$ is the phase error, $\delta \tau_{s}$ is the travel time error, and subscript $s$ denotes these errors are carried by the source side wave and linked to the local plane wave with wavenumber $\mathbf{k}_{s}$. Similarly, we have factor $a_{g} e^{i \omega \delta \tau_{g}}$ for the receiver side wave. According to Eq. (2.8), when mapped to the target, they change $A$ into

$$
A^{\prime}\left(\mathbf{x}, \mathbf{k}_{d}, \mathbf{x}_{s}, \mathbf{x}_{g}, \omega\right)=a\left(\mathbf{k}_{d}\right) \exp \left[i \varphi\left(\mathbf{k}_{d}\right)\right] A\left(\mathbf{x}, \mathbf{k}_{d}, \mathbf{x}_{s}, \mathbf{x}_{g}, \omega\right),
$$

where $a\left(\mathbf{k}_{d}\right)=a_{s} a_{g}$ is the amplitude error,

$$
\varphi\left(\mathbf{k}_{d}\right)=\frac{\left|\mathbf{k}_{d}\right| c_{0}(\mathbf{x}) \delta \tau}{2 \cos \left(\frac{O_{\mathbf{k}_{s}, \mathbf{k} g}}{2}\right)}
$$

is the phase error, $\delta \tau=\delta \tau_{s}+\delta \tau_{g}$, and $O_{\mathbf{k}_{s}, \mathbf{k}_{g}}$ is the opening angle between $\mathbf{k}_{s}$ and $\mathbf{k}_{g}$ (see e.g., Appendix B in Ref. [17]). Substituting $A^{\prime}$ in Eq. (2.10) for $A$ in Eq. (2.7), we see the factor $a\left(\mathbf{k}_{d}\right) \exp \left[i \varphi\left(\mathbf{k}_{d}\right)\right]$ introduces error to the $\mathbf{k}_{d}$ component of the wavenumber domain PSF. When transformed back to space domain, these errors affect the image. In particular, the phase error can directly destroy the zero phase image condition, causing serious defocusing. Because the phase error is proportional to $\left|\mathbf{k}_{d}\right|$, it will affect the high wavenumber components more seriously, causing the image to lose fine features first. Moreover, the mapping is not one-to-one. Two pairs of incident/scattered waves having different wavenumber magnitudes (i.e., different frequencies) and approaching the target from different directions may be mapped to the same wavenumber $\mathbf{k}_{d}$. Eqs. (2.1)-(2.11) reveal connections between phase errors generated in the shallow layer to the subsurface 
image, but highly complicated propagation and mapping relations prevent us from investigating this process analytically. We will turn to use numerical methods to investigate this process.

\section{Simulating shallow scattering using a random velocity model}

The shallow scattering layer is highly complicated, and it is difficult to directly characterize its properties. Random velocity models were used in seismology to investigate interactions between waves and scatterers of different scales. Wu and Aki [9] theoretically investigated elastic wave scattering in random media. Frankel and Clayton [18] discussed using finite difference methods to simulate seismic wave scattering in random velocity models. Sato et al. [10] comprehensively reviewed seismic wave propagation and scattering in a generally heterogeneous Earth. Random velocity models have been used to investigate Lg-wave energy partitions in random crustal waveguides [19-21], study Pnwave propagation in the random upper mantle [22], simulate anisotropic scattering in the reservoir [23], and calculate scattering in the near surface layer [24]. Major parameters for characterizing a random velocity model are power spectrum, correlation length and the rms velocity perturbation (see e.g., $[9,10,18])$. The type of random spectrum and the correlation length determine the distribution of heterogeneities versus the wavelength. Shown in Fig. 4 are three commonly used random spectra including Gaussian, exponential and self-similar $[10,18]$. Given the correlation length, the Gaussian random spectrum is dominated by low wavenumbers (large scale); the self-similar spectrum has the greatest high wavenumber (small scale) content; and the wavenumber content of exponential spectrum lies in between the Gaussian and self-similar spectra. The correlation length can be different along different directions, allowing control of the aspect ratio of scatterers. By properly choosing these parameters, we can build random velocity models to meet different purposes. Shown in Fig. 5 are sample velocity models for typical random spectra and parameters, where the model size is $2 \mathrm{~km} \times 2 \mathrm{~km}$, the background velocity is $3.5 \mathrm{~km} / \mathrm{s}$, the rms velocity perturbation is $10 \%$, and $a_{x}$ and $a_{z}$ are horizontal and vertical correlation lengths. From top to bottom are Gaussian, exponential and self-similar spectra. We see clearly how different parameters affect the shape of the heterogeneities. For example, models with $a_{x}>a_{z}$ are more suitable for sedimentary structures.

To investigate how errors of different scales in the migration velocity model can affect image quality, we first generated a 2-D acoustic model, which is $10 \mathrm{~km}$ along the horizontal and $5 \mathrm{~km}$ in depth. The model is composed of two layers, with its upper part being a $400 \mathrm{~m}$ thick random layer used to simulate shallow scattering. The layer has a low background velocity of $2000 \mathrm{~m} / \mathrm{s}$, to which is added a random velocity fluctuation. The fluctuation has an exponential random spectrum, the rms velocity perturbation is 10 $\%$, and the vertical and horizontal correlation lengths, $a_{x}$ and $a_{z}$, are both $200 \mathrm{~m}$. The lower layer is a homogeneous medium with a constant velocity of $3500 \mathrm{~m} / \mathrm{s}$. The density is assumed constant throughout the model. We use this model as the true velocity model. 


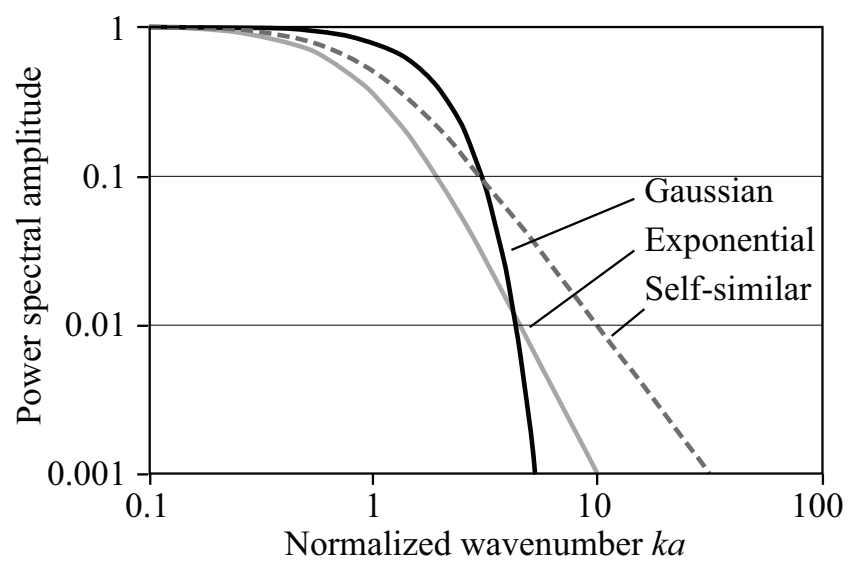

Figure 4: Three types of random spectra. The vertical coordinate is normalized amplitude, the horizontal coordinate is normalized wavenumber.
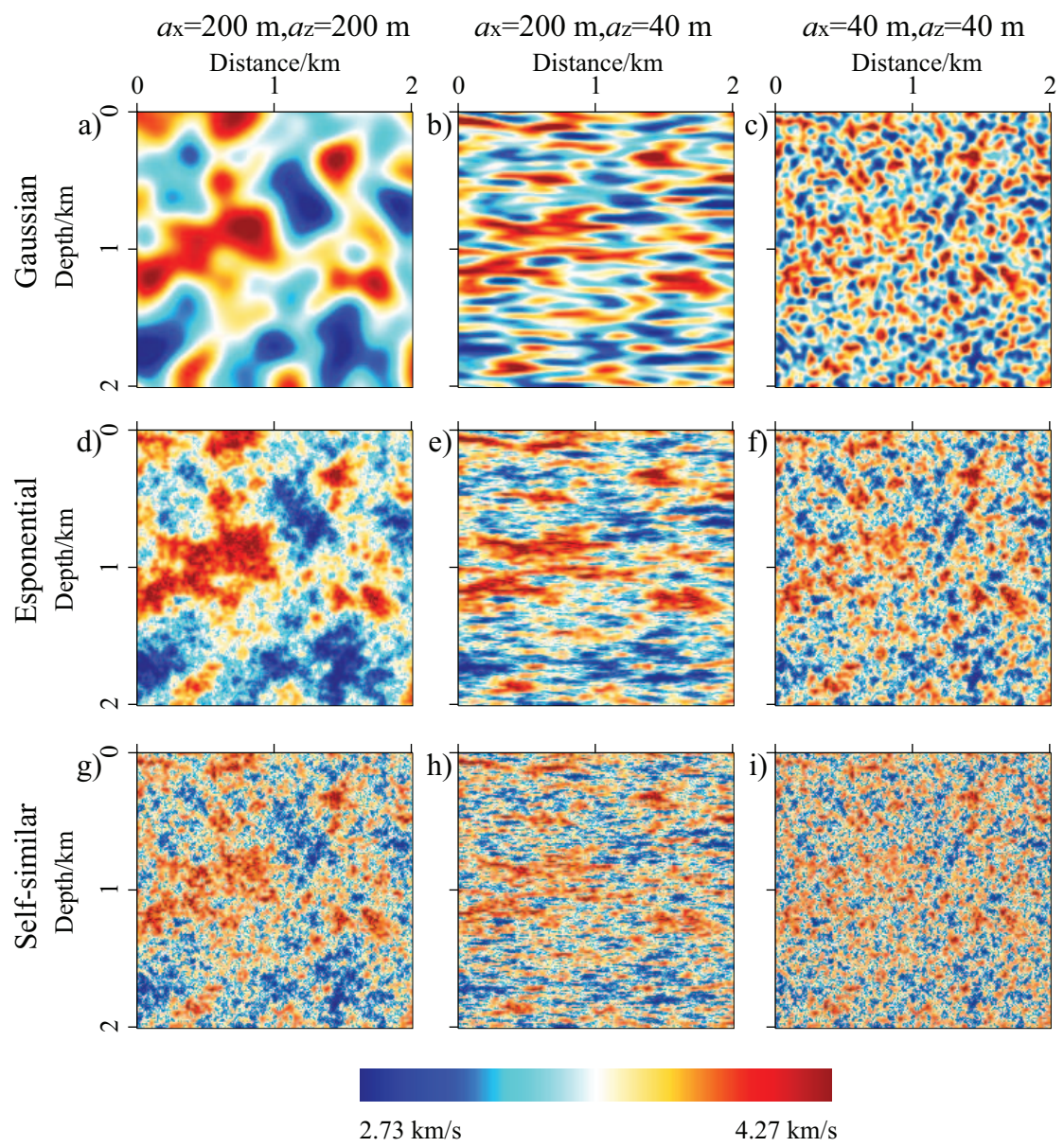

Figure 5: Typical random velocity models from three types of random spectra and different random parameters. 


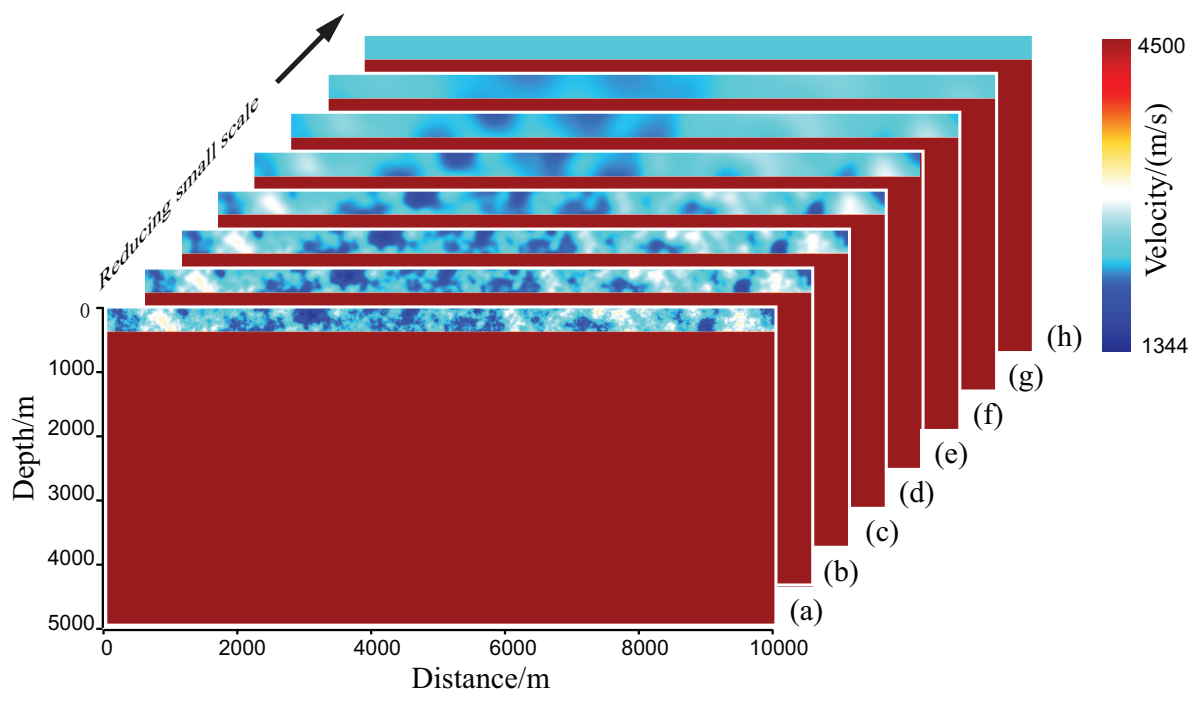

Figure 6: A group of two-layer models with different accuracies in the top layer. Model (a) in front is the true velocity model and model $(\mathrm{h})$ has a constant top layer. For models (b)-(g), their top layers successively lose accuracies from small to large scale.

To generate migration velocity models with different accuracies, the velocity in the top layer is smoothed using a two-dimensional Gaussian filter

$$
W(x, z)=B \exp \left\{-\left[\left(\frac{x}{\sigma_{x}}\right)^{2}+\left(\frac{z}{\sigma_{z}}\right)^{2}\right]\right\},
$$

where $\mathrm{x}$ and $\mathrm{z}$ are horizontal and vertical coordinates, $\sigma_{x}$ and $\sigma_{z}$ are characteristic scales controlling horizontal and vertical cutoff wavenumbers, and B is a normalization factor. Applying Eq. (3.1) with different characteristic scales to the true velocity model can generate migration velocity models with different accuracies in shallow heterogeneities. The smoothed models are shown in Fig. 6, where 6a is the original true velocity model without being filtered, $6 \mathrm{~h}$ has a constant top layer without any heterogeneity, and $6 \mathrm{~b}$ to $6 \mathrm{~g}$ are models filtered with $\sigma=\sigma_{x}=\sigma_{z}=30,50,100,200,300$ and $500 \mathrm{~m}$, respectively. From panels $6 \mathrm{a}$ to $6 \mathrm{~h}$, top layers successively lose their accuracies from small to large scale.

To further check differences among these models, their wavenumber spectra are illustrated in Fig. 7a with double logarithmic scales, where horizontal and vertical coordinates are the wavenumber and normalized spectral amplitude, and different curves are spectra with different $\sigma$. Illustrated in Fig. $7 \mathrm{~b}$ are velocities along the horizontal direction at $200 \mathrm{~m}$ depth, where the horizontal and vertical coordinates are distance and velocity. The unfiltered random model has the highest velocity perturbation and the richest high wavenumber content. As $\sigma$ increases, the random spectra shrink towards the low wavenumber, and the velocity models, compared to the true velocity, gradually lose their accuracies from small to large scales in the near-surface layer. We will use these models as 

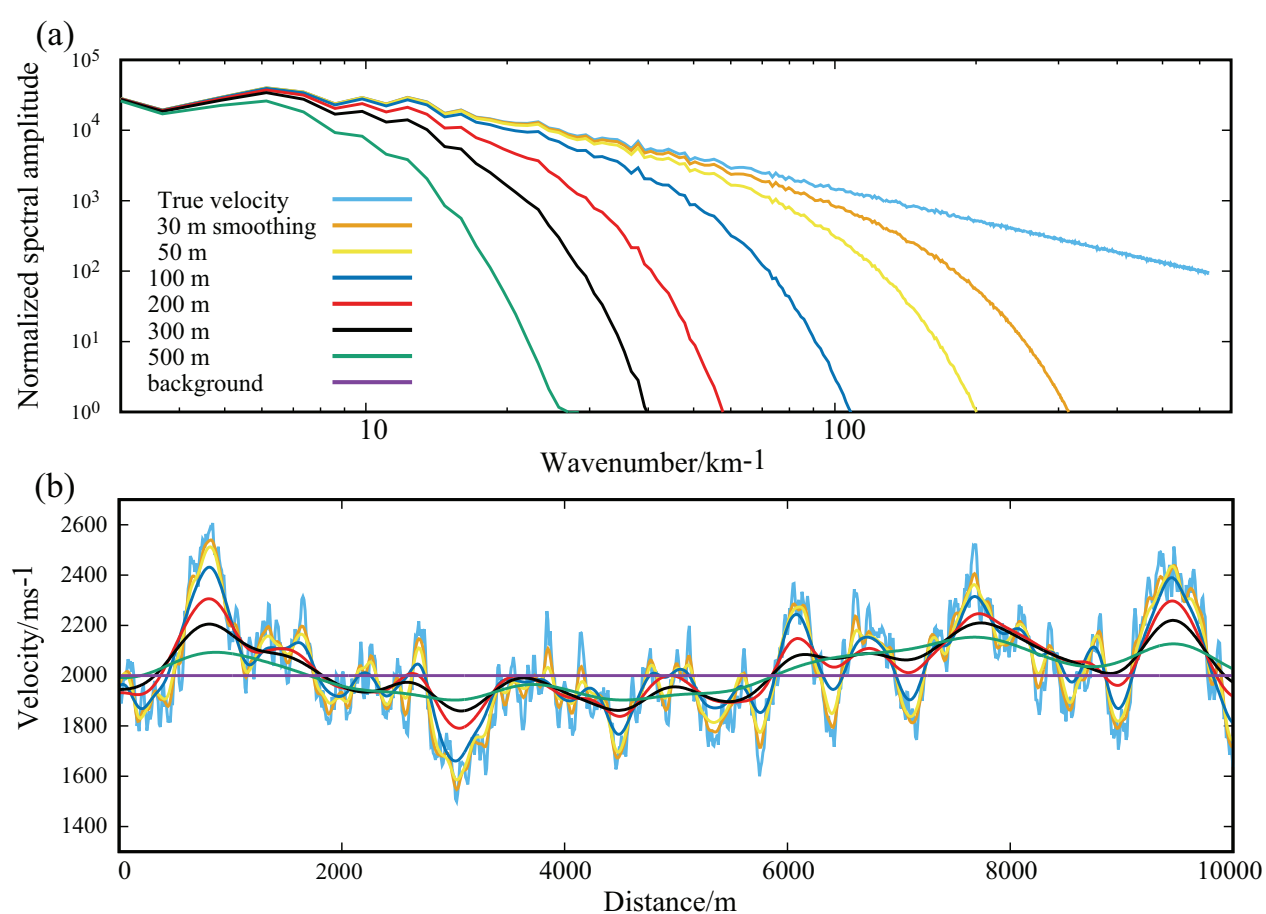

Figure 7: (a) Power spectra and (b) horizontal velocity variations in the shallow random layer. Different colors indicate models smoothed using different Gaussian filters.

migration velocity models to test how velocity errors of different scales affect the quality of depth image.

\section{Simulating shallow scattering using a random velocity model}

Seismic data are generated by waves passing through the real earth (or for synthetic data calculated in the true velocity model), and they carry information from heterogeneities of all scales. Ideally, during migration, time reversed seismic data are back propagated through the same velocity model, recovering the phase and amplitude of the original wavefield. However, when there are errors in the migration velocity model, the back propagated wavefields are distorted and lose correct amplitude and phase. We chose two basic situations, plane wave and spherical wave, to investigate how the velocity errors of different scales in the shallow layer can affect the reconstructed wavefield. Using the velocity shown in Fig. 6a as the true velocity model, upward-traveling plane wave passes through the shallow random layer, being recorded by receivers on the surface. We then time reverse the recorded wave, followed by downward extrapolation to reconstruct the original plane wavefield. During this process, we use models in Figs. 6a-6h as the migration velocity model and investigate relations between errors in the migration velocity and errors in the reconstructed wavefield. Fig. 8 captures the wavefield snapshot at the 

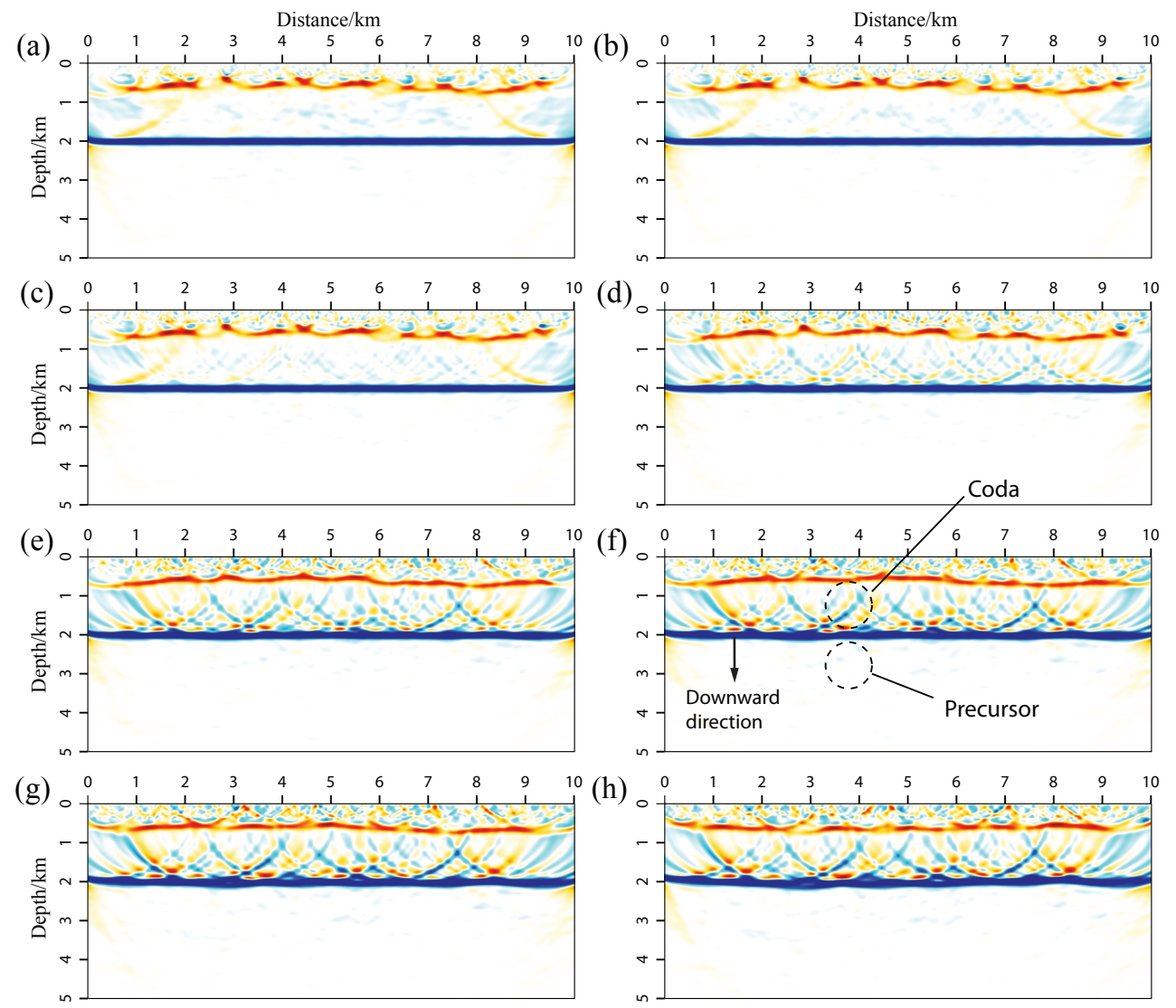

(f)

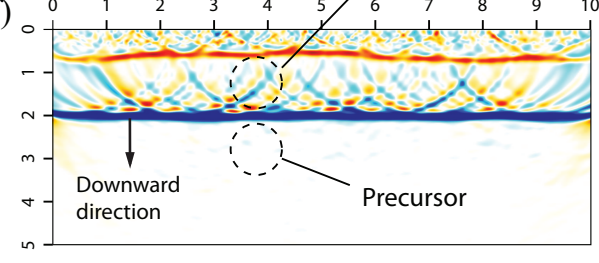

(h)

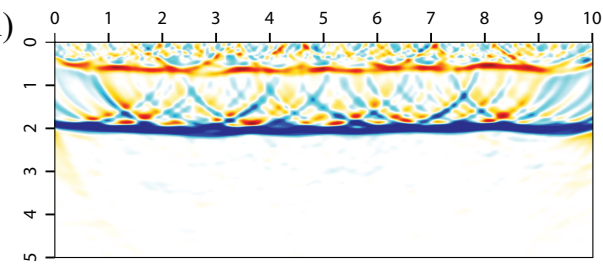

Figure 8: Snapshots for back propagated plane waves in migration velocity models with different accuracies. Wavefields in (a) to (h) are corresponding to velocity models in Figs. 6a to 6h, respectively.

time when the leading edge is $2 \mathrm{~km}$ deep, with $8 \mathrm{a}$ to $8 \mathrm{~h}$ corresponding to velocity models $6 \mathrm{a}$ to $6 \mathrm{~h}$. When using the true model $6 \mathrm{a}$ as the migration velocity model, the reconstructed wavefield has the highest accuracy. While models $6 \mathrm{~b}$ to $6 \mathrm{~h}$ are used, the results (Figs. $8 \mathrm{~b}$ to $8 \mathrm{~h}$ ) show increasingly distorted wavefields, in which travel time errors make the wave front biased from the original plane wave, and focusing/defocusing cause amplitude fluctuations. They form the amplitude and phase errors in Eq. (2.9). Some energy is moved from the primary wave to the coda wave behind the back propagation direction. Precursors, converted from time-reversed coda waves formed during the forward propagation, appear in front of the wavefront. The coda waves and precursors form the scattering noise. For a spherical wavefront, similar results can be seen in Fig. 9, with 9a to $9 \mathrm{~h}$ as back propagated wavefield snapshots corresponding to velocity models $6 \mathrm{a}$ to $6 \mathrm{~h}$. Due to the shrinking wavefront, the precursors are more severe than in the plane wave case. In both cases, we see that errors in shallow velocity models can seriously affect the accuracy of the reconstructed wavefields. As the migration velocity model loses more 

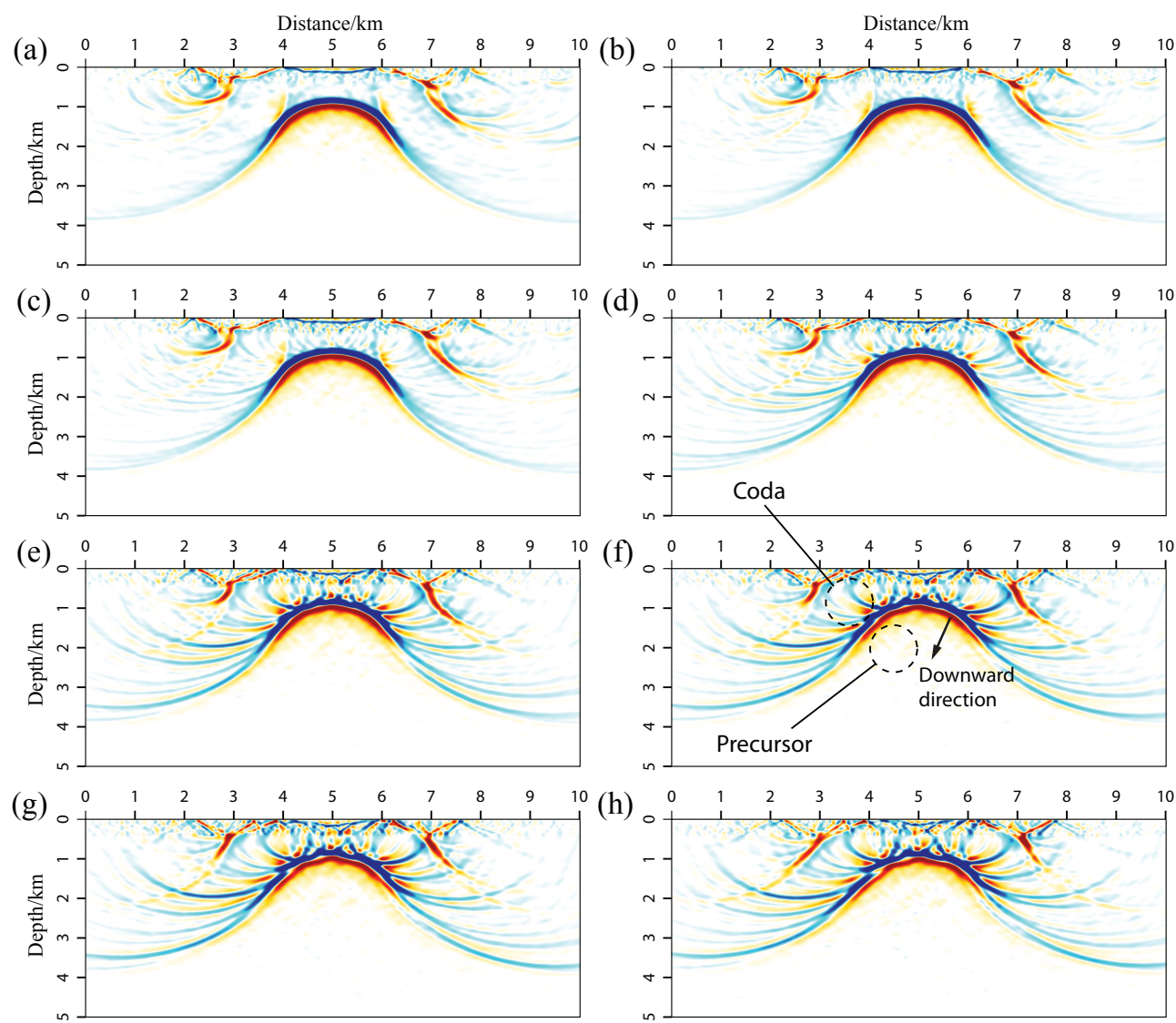

Figure 9: Similar to Fig. 8, except for spherical waves. Wavefields in (a) to (h) are corresponding to velocity models in Figs. 6a-6h, respectively.

intermediate and large scale components, the back propagated wavefront becomes more distorted. The accuracy of the migration velocity model, particularly its intermediate to large scale accuracy, is vital to reconstruct a high quality back propagated wavefield for imaging.

\section{The effects of shallow scattering on the point spread function}

From Eq. (2.3) or Eq. (2.5), the image can be expressed as a convolution between $R\left(\mathbf{x}, \mathbf{x}^{\prime}, \mathbf{x}^{\prime \prime}\right)$ and the velocity perturbation $M\left(\mathbf{x}^{\prime}\right)$. As a generalized image, the PSF contains all information about the resolution and defects of the imaging system. Investigating the behavior of PSFs allows study the influence of velocity model error on the quality of an imaging system. There are two different ways to calculate the PSF. With the first method, we could calculate Greens function $G_{D}$ using the true velocity model and $G_{M}$ using the migration velocity model with given errors, followed by using Eqs. (2.5)-(2.8) to calculate the PSF 

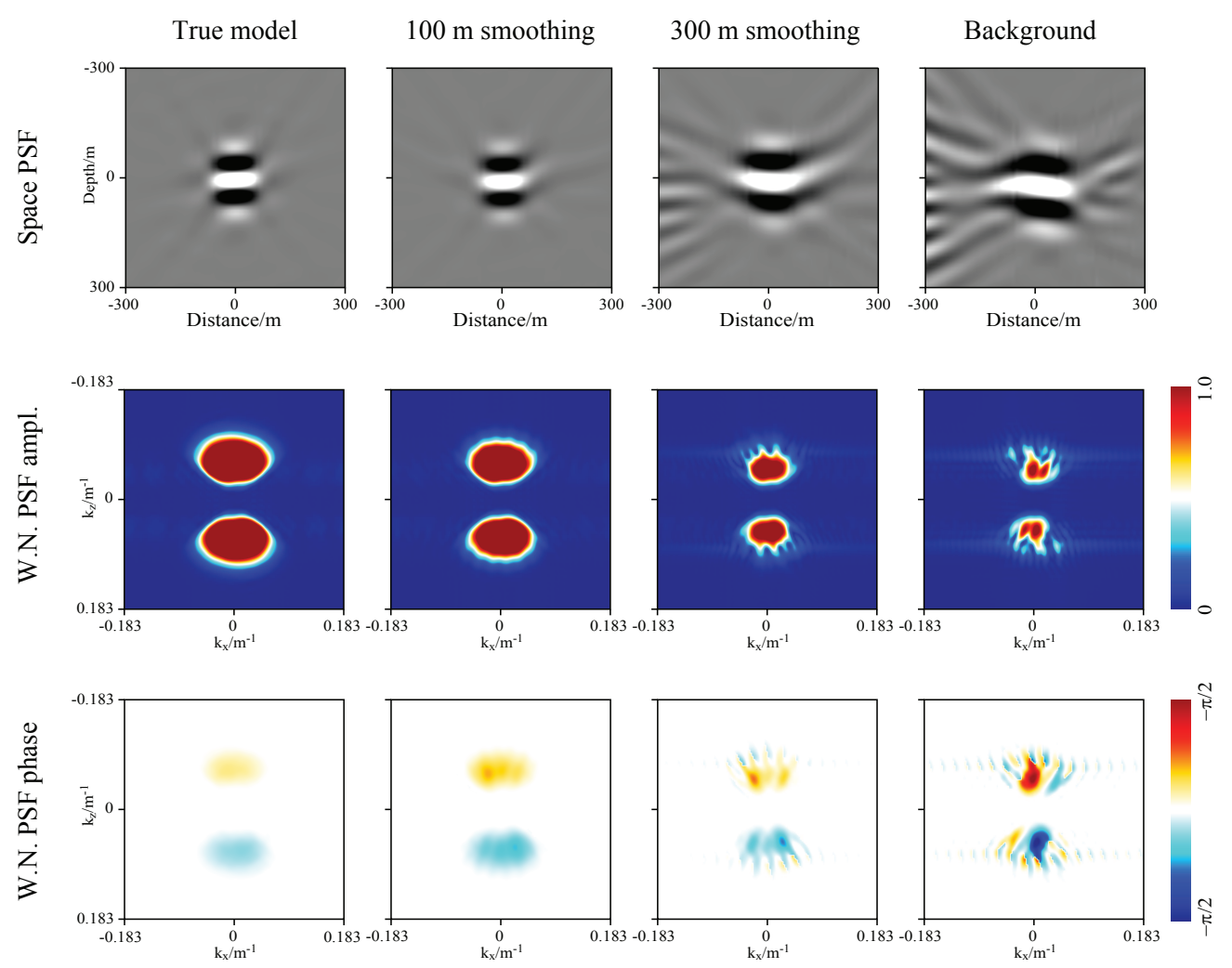

Figure 10: The PSFs calculated in different migration velocity models. The rows from top to bottom are space-domain PSFs and their amplitude and phase spectra in wavenumber domain. The four columns are calculated in the true velocity model (Fig. 6a), 100-m smoothed model (6d), 300-m smoothed model (6f), and the background model $(6 \mathrm{~h})$, respectively.

(Refs. [11,12]). According to Eq. (2.3), if the velocity perturbation is a sufficiently small scatterer, i.e., $M(\mathbf{x}) \approx \delta(\mathbf{x})$, where $\delta(\mathbf{x})$ is the Dirac delta function, we have $I(\mathbf{x}) \approx R(\mathbf{x})$. Alternatively, the PSF could be directly obtained by imaging a point scatterer $[24,25]$. The PSFs calculated from both methods are equivalent. We used the second method in following calculations.

We used the velocity model shown in Fig. 6a and implanted a point scatterer with 10 $\%$ velocity perturbation at $(5000 \mathrm{~m}, 3000 \mathrm{~m})$ to generate the synthetic data. The acquisition system was composed of 101 surface shots located between distances $2500 \mathrm{~m}$ and $7500 \mathrm{~m}$. Each source has 501 receivers with the source located at the center of the receiver array. The shot and receiver intervals were $50 \mathrm{~m}$ and $10 \mathrm{~m}$, and the source time function was a $16 \mathrm{~Hz}$ Ricker wavelet. Then, we used velocity models shown in Fig. 6 as the migration velocity models to calculate PSFs. The results are shown in Fig. 10, where three rows from top to bottom are space-domain PSFs and their amplitude and phase spectra in wavenumber domain. The four columns from left to right were calculated using the true velocity model (Fig. 6a), 100-m smoothed model (6d), 300-m smoothed model (6f), 
and the background velocity (6h), respectively. The space-domain PSF obtained using the true velocity model, even with strong scattering in the synthetic data, was well focused and unbiased. When using velocity models in Fig. $6 \mathrm{~d}$, the corresponding PSF was slightly affected but still well focused. When the smoothing radius increased to $300 \mathrm{~m}$, the near-surface layer started to lose middle to large scale velocity perturbations. The PSFs were seriously affected, and apparent artifacts develop. If we do not have any information on velocity perturbations in the near surface layer, the PSF has to be calculated using the constant background velocity, and its focusing is severely degraded.

We further analyze effects of shallow velocity errors on the amplitude and phase spectra of PSFs in the wavenumber domain. The results are shown in the second and third rows in Fig. 10. The amplitude spectra are normalized to unity and the phase spectra are normalized to between $\pm \pi / 2$. For spectrum obtained using the true velocity model, the amplitude spectrum is rich in high-wavenumber content, indicating the highest spatial resolution. The phase spectrum is close to zero in a broad wavenumber domain, indicating the zero phase image condition is met even for relatively high wavenumbers. As the near surface layer gradually loses accuracies from small- to large-scale, the amplitude spectra continually shrink towards the low-wavenumber and the phase error increases and damages the zero phase image condition (columns 2 to 4 ). When the phase error approaches $\pm \pi / 2$, constructive interference becomes impossible. As pointed out in Eqs. (2.7) to (2.11), the phase and travel time errors are generated in the near surface layer, and then carried to the target by the propagation and mapping to affect the PSF. Numerical calculations clearly reveal this, even with complex processes involved.

\section{Influence of migration velocity errors on the depth image}

To investigate the effect of near surface scattering on the seismic image, we constructed velocity models which included both a shallow random velocity layer and deep reflectors. The model size, acquisition geometry and source parameters were the same as those used before. A similar random velocity model was used for the shallow layer. The lower part of the model was composed of 5 layers (as in Fig. 11a). Four sets of synthetic data were calculated for models with their top layers having rms perturbations of $0 \%, 5 \%$, $10 \%$, and $15 \%$, but their random shape functions were the same as in Fig. 11a. Shown in Fig. 12 are shot gathers calculated in these models. For the three rows from top to bottom, the shot locations are at distances $2.5,5.0$, and $7.5 \mathrm{~km}$, respectively. For the four columns from left to right, the rms velocity perturbations are $0 \%, 5 \%, 10 \%$, and $15 \%$, respectively. As can be seen in the left column in Fig. 12, without shallow scattering ( $\mathrm{rms}=0 \%$ ), the primary and head waves are clearly seen. Reflections from deep interfaces can be clearly identified and their phases are highly coherent. With the increase of rms perturbations in the random layer, strong coda waves are formed, making the signal-to-noise ratio low. The reflection signals become incoherent. When the rms velocity fluctuations increase to $10 \%-15 \%$ (columns 3 and 4 ), the shot gathers are dominated by scattering noise and 


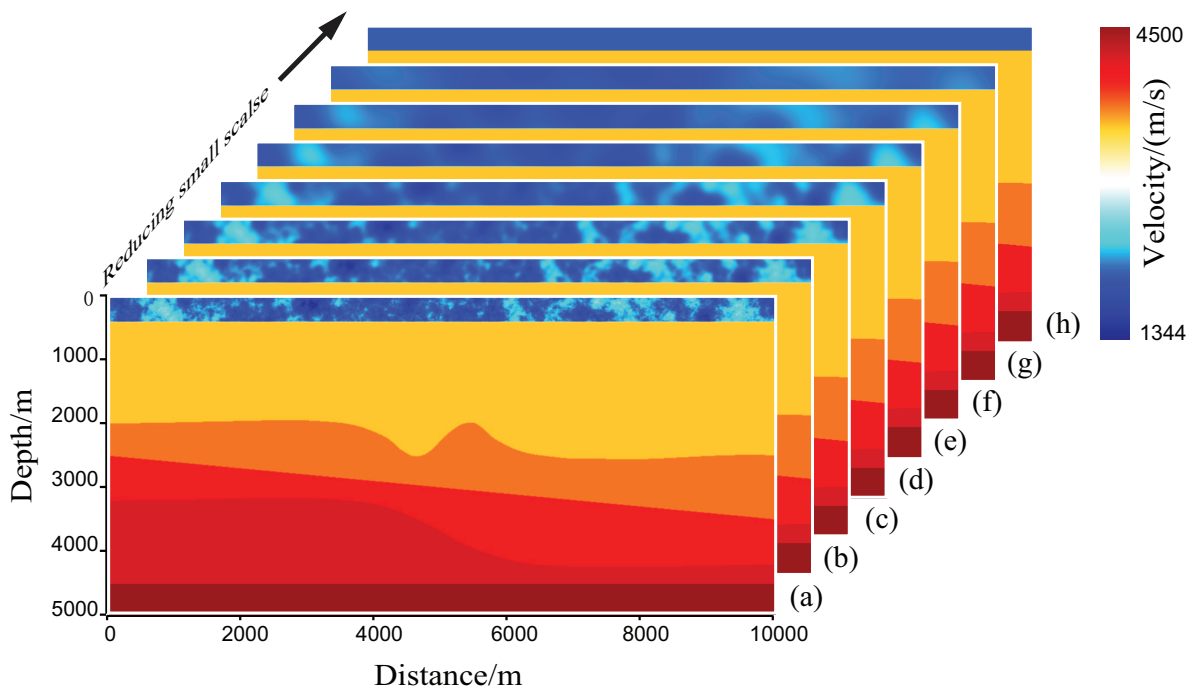

Figure 11: Similar to those shown in Fig. 6, except their lower parts are replaced by a layered structure.

reflection signals can hardly be identified. The synthetic data appear like the real data shown in Fig. 1, except here we used the acoustic model.

As the first test, assuming we do not have detailed information in the near surface layer, the velocity model with a constant top layer, Fig. 11h, is used for migration. Depth images are calculated using RTM, and are shown in Fig. 13, where 13a-13d are from synthetic data sets with rms velocity perturbations of $0 \%, 5 \%, 10 \%$, and $15 \%$, respectively. In Fig. 13a, since there is no near-surface scattering in the true velocity model $(\mathrm{rms}=0)$, the deep reflectors can be perfectly imaged. When the rms velocity perturbations in the true velocity reach to $5 \%$, the depth image is only slightly affected. However, if rms velocity fluctuations in the true velocity model reach to $10 \%-15 \%$, the image encountered serious problem. Recall the results in Section 4, where there are serious phase errors in reconstructed wavefield. The phase error is the primary reason responsible for the image problem. These results resemble the situation encountered in practice.

By investigating the behavior of PSFs in previous sections, we see that if certain intermediate to large scale velocity perturbations can be built into the migration velocity, the amplitude and phase errors in PSF can be significantly reduced. To verify this in the imaging process, we chose the model shown in Fig. 11a $(\mathrm{rms}=10 \%)$ as the true velocity model to calculate a synthetic data set. To generate migration velocity models with different accuracies, we follow the previous sections and apply Eq. (3.1) to smooth the shallow velocity perturbations in the true velocity model. Resulting models are shown in Fig. 11, where 11a is the true velocity model without being smoothed, $11 \mathrm{~h}$ is the model without perturbations in the top layer, and 11b-11g are models smoothed with $\sigma=30$, 50, 100, 200, 300 and $500 \mathrm{~m}$, respectively. From panels 11a to 11h, top layers gradually lose their accuracies from small to large scale. Then, RTM was performed using these 


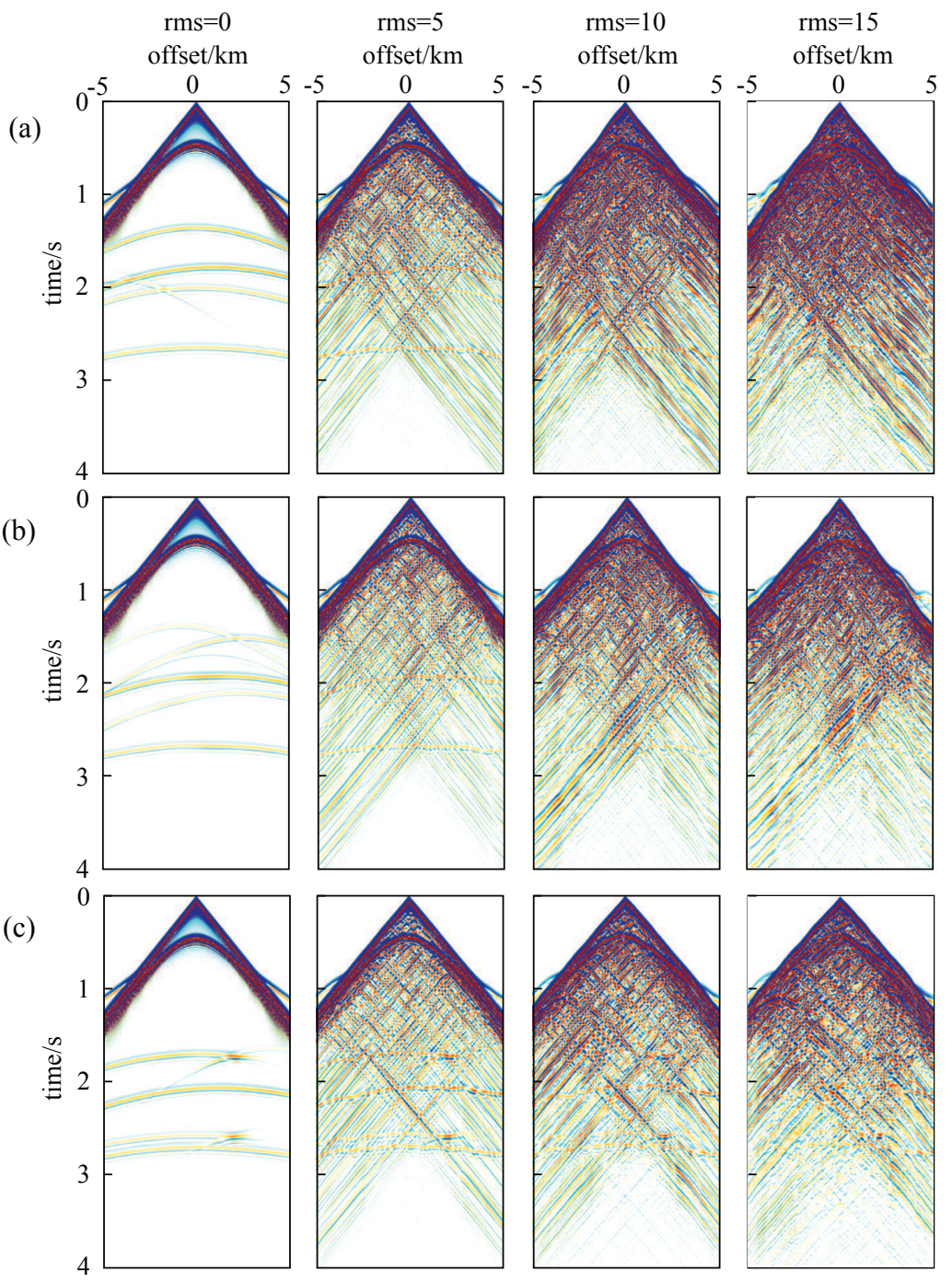

Figure 12: Shot gathers calculated in velocity models with different near surface random layers. For the four columns from left to right, the rms random velocity perturbations are $0 \%, 5 \%, 10 \%$, and $15 \%$, respectively. For the three rows from top to bottom, the shot locations are at distances $2.5,5.0$, and $7.5 \mathrm{~km}$, respectively.

models as the migration velocity. No first arrival muting or demultiple was used. The resulted images shown in Figs. 14a to 14h were calculated by using models 11a to $11 \mathrm{~h}$ as the migration velocity.

Fig. 14a is the image calculated by using the true model as the migration velocity. The deep reflectors are properly imaged. The results indicate that, even with strong scattering noise and serious phase fluctuations in the data, a correct migration velocity model can repair the wavefield during the back propagation, and a depth image of reasonable quality can be obtained. At the other extreme, if the background velocity is used, the 

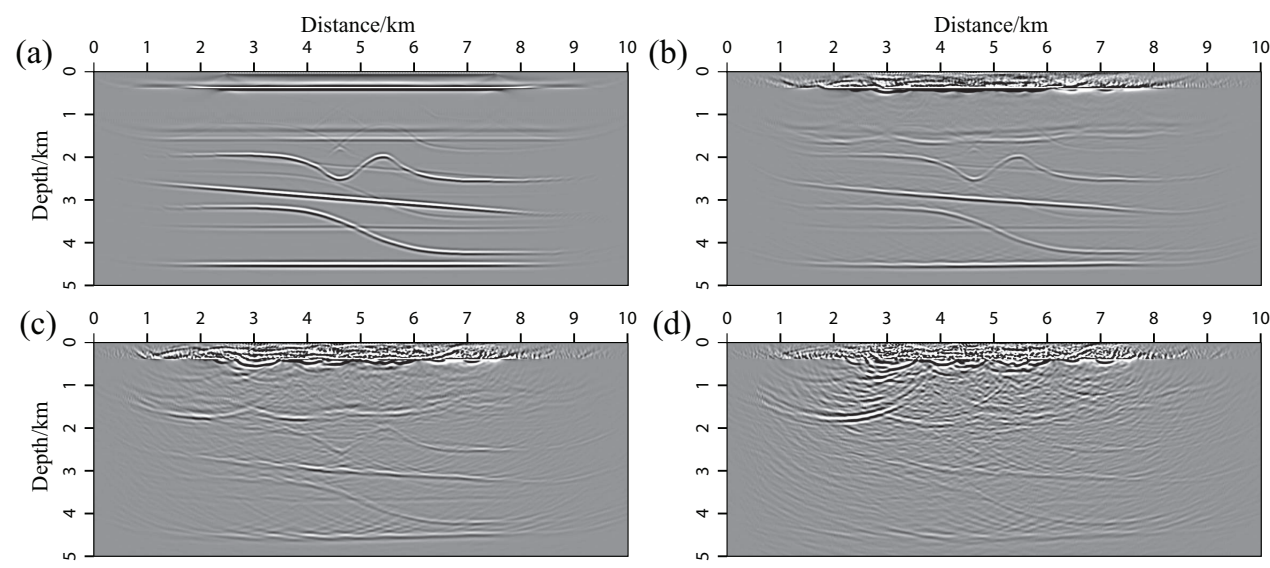

Figure 13: RTM images. The synthetic data sets are calculated in models with different rms velocity perturbations in shallow layers, with (a) $0 \%$, (b) $5 \%$, (c) $10 \%$, and (d) $15 \%$, respectively. The images are calculated in migration velocity models with a constant top layer.
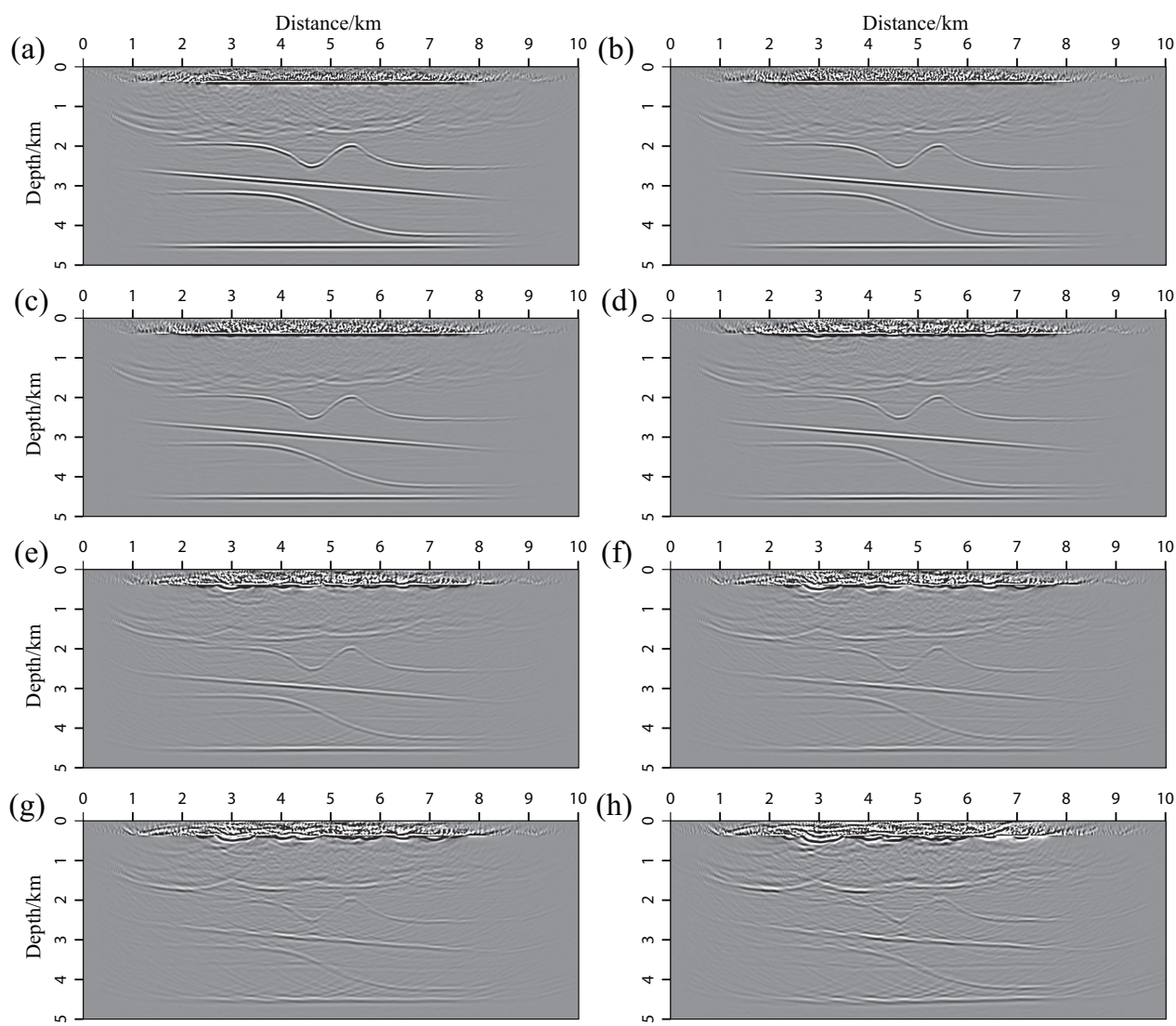

Figure 14: Depth images calculated using different migration velocity models shown in Fig. 11, where panels (a) to (h) corresponding to velocity models shown in 11 a to $11 \mathrm{~h}$. 
image quality is poor (Fig. 14h). Although it is difficult to create a migration velocity model including every detail, it is possible to retrieve certain intermediate to large scale perturbations and build them into the migration velocity to improve the image quality. By investigating Figs. 14b-14g, when the smoothing factor $\sigma \leq 100 \mathrm{~m}$, the image is only slightly affected. As $\sigma$ reaches $300 \mathrm{~m}$ (Fig. 14f), the image is apparently affected. With even larger $\sigma$, images are seriously affected. These results indicate that velocity perturbations of intermediate to larger scales are important for high quality imaging. By combining techniques such as the near-surface high-precision velocity model building, full waveform inversion in near surface layer, wave equation based static correction, or other more sophisticated methods [26-28] , it is possible to obtain a high-precision near surface velocity model just for the top $0.5-1.0 \mathrm{~km}$ to meet the required accuracy. On the other hand, the small scale velocity errors in the migration model do not contribute much to the phase error in the image. Considering retrieving such small-scale velocity structures is impractical, their effect may be removed using a filtering or stacking method.

\section{Discussions and conclusion}

We studied how shallow heterogeneities affect the seismic image. A random velocity model was used to simulate the shallow velocity structure, and reverse time migration was used to connect the errors in the migration velocity model to the errors in the depth image. In addition to the commonly known scattering noise which lowers the signal to noise ratio of the data, the phase errors generated by the intermediate to large scale heterogeneities due to multiple forward scattering is another important source of degraded image. The behavior of seismic wave scattering is closely related to the wavelength and the size of the scatterer. To investigate how these characteristic scale lengths affect the imaging process, we generated migration velocity models with accuracies of different scales, and studied their effects in different aspects including data noise, wave front distortion and coda/ precursor in downward extrapolated wavefield, the amplitude and phase errors in point spread function, and the quality of depth image. Our investigation indicated that, if high resolution velocities down to 100-300 m scale can be built into the near surface migration velocity model, the phase errors can be largely removed and the image quality can be significantly improved. It is possible using current acquisition density for imaging and the corresponding near-surface velocity inversion techniques to build velocity models with such accuracy. Most existing methods attempt to suppress scattering through stacking and filtering, which should be effective for reducing the random noise. However, coherent phase errors should be compensated using high precision migration velocity mode. The current numerical simulations were conducted in a constant density acoustic media under the 2D geometry, and no free surface condition was used. Further studies may be extended to more realistic situations, e.g., elastic model, 3D scattering, and using random velocity parameters based on local near surface velocity structures. 


\section{Acknowledgments}

The authors wish to thank Dr. Samuel Gray and another anonymous reviewer for their comments which greatly improved the manuscript of this paper.

\section{References}

[1] Z. Xia, S.H. Zhang and X.J. Wang, Discussion on near-surface characters and structures in complex area in western China, Oil Geophys. Prospect. (in Chinese), 38 (2003), 414-424.

[2] C. Gerea, J.-M. Mougenot and F. Clement, Seismic imaging in thrust-belts with rugged topography a 3D modeling approach, 81st Annual International Meeting, SEG, Expanded Abstracts (2011), 2881-2885.

[3] X. Zhu, K. Wallace, Q. Zhu and R. Hofer, Scattering effect on shallow gas-obscured zone imaging in Bohai PL19-3 area, Geophysics, 77 (2012), B43B53.

[4] C. Regone, J. Stefani, P. Wang, C. Gerea, G. Gonzalez, and M. Oristaglio, Geologic model building in SEAM Phase II - Land seismic challenges, The Leading Edge, September (2017), 738-749.

[5] D.L. Tang, X.W. Cai, Y.Q. He and H.X. Ning, Source and receiver arrays for prestack migration, Oil Geophys. Prospect. (in Chinese), 49 (2014), 1034-1038.

[6] Y.G. Wu, H.X. Ning, H.L. Wang and J. Hu, Application of a wide line and geophone continuous array seismic acquisition technique in the mountainous YingXiongling area of the Qaidam basin, 82th Annual International Meeting, SEG, Expanded Abstracts, (2012), 1-5.

[7] A. Bakulin, P. Golikov, M. Dmitriev, V. Dolgov, P. Leger, D. Neklyudov, Application of supergrouping to land seismic data in desert environment, 86th Annual International Meeting, SEG, Expanded Abstracts (2016), 4649-4653.

[8] H.X. Ning, Y.G. Wu, D.L. Tang, G.Y. Li, G.S. Li. A 3-D Seismic prospecting integration solution in a complex mountainous region, western qaidam basin. 76th Annual International Conference and Exhibition, EAGE, Extended Abstracts, (2014) We P04 08.

[9] R.S. Wu and K. Aki, Elastic wave scattering by a random medium and the small scale inhomogeneities in the lithosphere, J. Geophys. Res., 90 (1985), 10261-10273.

[10] H. Sato, M.C. Fehler and T. Maeda, Seismic Wave Propagation and Scattering in the Heterogenous Earth, (2012), Springer-Verlag Berlin Heidelberg, ISBN 978-3-642-23028-8.

[11] X.B. Xie, R.S. Wu, M. Fehler and L. Huang, Seismic resolution and illumination. A waveequation based analysis. 75th Annual International Meeting, SEG, Expanded Abstracts (2005), 18621865.

[12] Z. Yan and X.B. Xie, Full-wave seismic illumination and resolution analyses: A Poyntingvector-based method, Geophysics, 81 (2016), S447-S458.

[13] J. Chen, and G. Schuster, Resolution limits of migrated images: Geophysics, 64, (1999), 10461053.

[14] L.J. Gelius, I. Lecomte and H. Tabti, Analysis of the resolution function in seismic prestack depth imaging: Geophys. Prospect., 50 (2002), 505-515.

[15] R.L. Gibson, Jr. and C. Tzimeas, Quantitative measures of image resolution for seismic survey design, Geophysics, 67 (2002), 18441852.

[16] I. Lecomte, Resolution and illumination analyses in PSDM: A ray-based approach: The Leading Edge, 27 (2008), 650663. 
[17] X.B. Xie and H. Yang, The finite-frequency sensitivity kernel for migration residual moveout and its applications in migration velocity analysis, Geophysics, 73 (2008), S241S249.

[18] A. Frankel and R.W. Clayton, Finite difference simulations of seismic scattering implications for the propagation of short-period seismic waves in the crust and models of crustal heterogeneity, J. Geophys. Res., 91 (1986), 6465-6489.

[19] X.B. Xie and T. Lay, The excitation of explosion Lg, a finite-difference investigation. Bull. Seis. Soc. Am., 84 (1994), 324-342.

[20] R.S. Wu, S. Jin and X.B. Xie, Energy partition and attenuation of Lg waves by numerical simulations using screen propagators, Phys, Earth Planet. Inter., 120 (2000), 227-244.

[21] X.B. Xie, Z. Ge and T. Lay, Investigating explosion source energy partitioning and Lg-wave excitation using a finite-difference plus slowness analysis method. Bull. Seism. Soc. Am., 95 (2005), 2412-2427.

[22] X.B. Xie and T. Lay, Frequency-dependent effects of 2D random velocity heterogeneities in the mantle lid on Pn geometric spreading, Bull. Seism. Soc. Am., 107 (2017), 482-488.

[23] X.B. Xie, Seismic wave scattering in 3D random media: A finite-difference simulation and slowness domain analysis. 83rd Annual International Meeting, SEG, Expanded Abstracts, (2013), 3428-3431.

[24] B. Chen, H.X. Ning, and X.B. Xie, 2016, Investigating the effect of shallow scatterings from small scale near surface heterogeneities on seismic imaging: A resolution analysis based method, Chin. J. Geophys., 59 (2016), 1762-1775.

[25] J. Cao, Resolution/illumination analysis and imaging compensation in 3D dip-azimuth domain. 83rd Annual International Meeting, SEG, Expanded Abstracts (2013), 39313936.

[26] X. Zhu, P. Valasek1, B. Roy, S. Shaw, J. Howell, S. Whitney, N.D. Whitmore and P. Anno, Recent applications of turning-ray tomography, Geophysics, 73 (2008), VE243VE254.

[27] W. Wang, P. Chen and S. Holbrook. Near-surface adjoint tomography based on the discontinuous Galerkin method. 86th Annual International Meeting, SEG Expanded Abstracts, (2016), 2387-2392.

[28] J.T. Etgen, I. Ahmed, and M. Zhou, Seismic Adaptive Optics. 84th Annual International Meeting, SEG, Expanded Abstracts (2014), 4411-4415. 